\title{
Springer and the
}

\section{environment}

At Springer we firmly believe that an international science publisher has a special obligation to the environment, and our corporate policies consistently reflect this conviction.

We also expect our business partners paper mills, printers, packaging manufacturers, etc. - to commit themselves to using materials and production processes that do not harm the environment. The paper in this book is made from low- or no-chlorine pulp and is acid free, in conformance with international standards for paper permanency.

\section{Springer}


Printing: Druckhaus Beltz, Hemsbach

Binding: Buchbinderei Schäffer, Grünstadt 


\title{
Lecture Notes in Physics
}

\author{
For information about Vols. 1-483 \\ please contact your bookseller or Springer-Verlag
}

Vol. 484: L. Schimansky-Geier, T. Pöschel (Eds.), Stochastic Dynamics. XVIII, 386 pages. 1997.

Vol. 485: H. Friedrich, B. Eckhardt (Eds.), Classical, Semi-classical and Quantum Dynamics in Atoms. VIII, 341 pages. 1997.

Vol. 486: G. Chavent, P. C. Sabatier (Eds.), Inverse Problems of Wave Propagation and Diffraction. Proceedings, $1996 . X V, 379$ pages. 1997.

Vol. 487: E. Meyer-Hofmeister, H. Spruik (Eds.), Accretion Disks - New Aspects. Proceedings, 1996. XIII, 356 pages. 1997.

Vol. 488: B. Apagyi, G. Endrédi, P. Lévay (Eds.), Inverse and Algebraic Quantum Scattering Theory. Proceedings, 1996. XV, 385 pages. 1997.

Vol. 489: G. M. Simnett, C. E. Alissandrakis, L. Vlahos (Eds.), Solar and Heliospheric Plasma Physics. Proceedings, 1996. VIII, 278 pages. 1997.

Vol. 490: P. Kutler, J. Flores, J.-J. Chattot (Eds.), Fifteenth International Conference on Numerical Methods in Fluid Dynamics. Proceedings, 1996. XIV, 655 pages. 1997.

Vol. 491: O. Boratav, A. Eden, A. Erzan (Eds.), Turbulence Modeling and Vortex Dynamics. Proceedings, 1996. XII, 245 pages. 1997.

Vol. 492: M. Rubí, C. Pérez-Vicente (Eds.), Complex Behaviour of Glassy Systems. Proceedings, 1996. IX, 467 pages. 1997.

Vol. 493: P. L. Garrido, J. Marro (Eds.), Fourth Granada Lectures in Computational Physics. XIV, 316 pages. 1997.

Vol. 494: J. W. Clark, M. L. Ristig (Eds.), Theory of Spin Lattices and Lattice Gauge Models. Proceedings, 1996. XI, 194 pages. 1997.

Vol. 495: Y. Kosmann-Schwarzbach, B. Grammaticos, K.M. Tamizhmani (Eds.), Integrability of Nonlinear Systems. VII, 380 pages. 1997.

Vol. 496: F. Lenz, H. Grießhammer, D. Stoll (Eds.), Lectures on QCD. VII, 483 pages. 1997.

Vol. 497: J. P. Greve, R. Blomme, H. Hensberge (Eds.), Stellar Atmospheres: Theory and Observations. VIII, 352 pages. 1997

Vol. 498: Z. Horváth, L. Palla (Eds.), Conformal Field Theories and Integrable Models. Proceedings, 1996. X, 251 pages. 1997.

Vol. 499: K. Jungmann, J. Kowalski, I. Reinhard, F. Träger (Eds.), Atomic Physics Methods in Modern Research, IX, $44^{8}$ pages. 1997.

Vol. 500: D. Joubert (Ed.), Density Functionals: Theory and Applications, XVI, 194 pages. 1998.

Vol. 501: J. Kertész, I. Kondor (Eds.), Advances in Computer Simulation. VIII, 166 pages. 1998.

Vol. 502: H. Aratyn, T. D. Imbo, W.-Y. Keung, U. Sukhatme (Eds.), Supersymmetry and Integrable Models. Proceedings, 1997. XI, 379 pages. 1998.

Vol. 503: J. Parisi, S. C. Müller, W. Zimmermann (Eds.), A Perspective Look at Nonlinear Media. From Physics to Biology and Social Sciences. VIII, 372 pages. 1998.

Vol. 504: A. Bohm, H.-D. Doebner, P. Kielanowski(Eds.), Irreversibility and Causality. Semigroups and Rigged Hilbert Spaces. XIX, 385 pages. 1998.

Vol. 505: D. Benest, C. Froeschlé (Eds.), Impacts on Earth. XVII, 223 pages. 1998.

Vol. 506: D. Breitschwerdt, M. J. Freyberg, J. Trümper (Eds.), The Local Bubble and Beyond. Proceedings, 1997. XXVIII, 603 pages. 1998.

Vol. 507: J. C. Vial, K. Bocchialini, P. Boumier (Eds.), Space Solar Physics. Proceedings, 1997. XIII, 296 pages. 1998.

Vol. 508: H. Meyer-Ortmanns, A. Klümper (Eds.), Field Theoretical Tools for Polymer and Particle Physics. XVI, 258 pages. 1998.

Vol. 509: J. Wess, V. P. Akulov (Eds.), Supersymmetry and Quantum Field Theory. Proceedings, 1997. XV, 405 pages. 1998.

Vol. 510: J. Navarro, A. Polls (Eds.), Microscopic Quantum Many-Body Theories and Their Applications. Proceedings, 1997. XIII, 379 pages. 1998.

Vol. 511: S. Benkadda, G. M. Zaslavsky (Eds.), Chaos, Kinetics and Nonlinear Dynamics in Fluids and Plasmas. Proceedings, 1997. VIII, 438 pages. 1998.

Vol. 512: H. Gausterer, C. Lang (Eds.), Computing Particle Properties. Proceedings, 1997. VII, 335 pages. 1998.

Vol. 513: A. Bernstein, D. Drechsel, T. Walcher (Eds.), Chiral Dynamics: Theory and Experiment. Proceedings, 1997. IX, 394 pages. 1998.

Vol. 514: F.W. Hehl, C. Kiefer, R.J.K. Metzler, Black Holes: Theory and Observation. Proceedings, 1997. XV, 519 pages. 1998.

Vol. 515: C.-H. Bruneau (Ed.), Sixteenth International Conference on Numerical Methods in Fluid Dynamics. Proceedings. XV, 568 pages. 1998.

Vol. 516: J. Cleymans, H. B. Geyer, F. G. Scholtz (Eds.), Hadrons in Dense Matter and Hadrosynthesis. Proceedings, 1998. XII, 253 pages. 1999.

Vol. 517: Ph. Blanchard, A. Jadczyk (Eds.), Quantum Future. Proceedings, 1997. X, 244 pages. 1999.

Vol. 518: P. G. L. Leach, S. E. Bouquet, J.-L. Rouet, E. Fijalkow (Eds.), Dynamical Systems, Plasmas and Gravitation. Proceedings, 1997. XII, 397 pages. 1999.

Vol. 519: A. Pẹkalski, K. Sznajd-Weron (Eds.), Anomalous Diffusion. From Basics to Applications. Proceedings, 1998. XVIII, 378 pages. 1999.

Vol. 520: J. A. van Paradijs, J. A. M. Bleeker (Eds.), XRay Spectroscopy in Astrophysics. EADN School X. Proceedings, 1997. XV, 530 pages. 1999.

Vol. 521: L. Mathelitsch, W. Plessas (Eds.), Broken Symmetries. Proceedings, 1998. VII, 299 pages. 1999.

Vol. 523: B. Wolf, O. Stahl, A.W. Fullerton (Eds.), Variable and Non-spherical Stellar Winds in Luminous Hot Stars. Proceedings, 1998. XX, 424 pages. 1999. 


\section{Monographs}

\section{For information about Vols. 1-10 please contact your bookseller or Springer-Verlag}

Vol. m 11: A. D. Yaghjian, Relativistic Dynamics of a Charged Sphere. XII, 115 pages. 1992.

Vol.m 12: G. Esposito, Quantum Gravity, Quantum Cosmology and Lorentzian Geometries. Second Corrected and Enlarged Edition. XVIII, 349 pages. 1994

Vol. I3: $_{13}$ M. Klein, A. Knauf, Classical Planar Scattering by Coulombic Potentials. V, 142 pages. 1992.

Vol. $m$ 14: A. Lerda, Anyons. XI, 138 pages. 1992.

Vol. m 15: N. Peters, B. Rogg (Eds.), Reduced Kinetic Mechanisms for Applications in Combustion Systems. $\mathrm{X}, 360$ pages. 1993 .

Vol. m 16: P. Christe, M. Henkel, Introduction to Conformal Invariance and Its Applications to Critical Phenomena. XV, 260 pages. 1993.

Vol. $m$ 17: M. Schoen, Computer Simulation of Condensed Phases in Complex Geometries. X, 136 pages. 1993.

Vol. m 18: H. Carmichael, An Open Systems Approach to Quantum Optics. X, 179 pages. 1993.

Vol. m 19: S. D. Bogan, M. K. Hinders, Interface Effects in Elastic Wave Scattering. XII, 182 pages. 1994.

Vol. m 20: E. Abdalla, M. C. B. Abdalla, D. Dalmazi, A. Zadra, 2D-Gravity in Non-Critical Strings. IX, 319 pages. 1994.

Vol. m 21: G. P. Berman, E. N. Bulgakov, D. D. Holm, Crossover-Time in Quantum Boson and Spin Systems. XI, 268 pages. 1994.

Vol. $m$ 22: M.-O. Hongler, Chaotic and Stochastic Behaviour in Automatic Production Lines. V, 85 pages. 1994.

Vol. $m$ 23: V. S. Viswanath, G. Müller, The Recursion Method. X, 259 pages. 1994.

Vol. m 24: A. Ern, V. Giovangigli, Multicomponent Transport Algorithms. XIV, 427 pages. 1994.

Vol. m 25: A. V. Bogdanov, G. V. Dubrovskiy, M. P. Krutikov, D. V. Kulginov, V. M. Strelchenya, Interaction of Gases with Surfaces. XIV, 132 pages. 1995.

Vol. m 26: M. Dineykhan, G. V. Efumov, G. Ganbold, S. N. Nedelko, Oscillator Representation in Quantum Physics. IX, 279 pages. 1995.

Vol. m 27: J. T. Ottesen, Infinite Dimensional Groups and Algebras in Quantum Physics. IX, 218 pages. 1995.

Vol. m 28: O. Piguet, S. P. Sorella, Algebraic Renormalization. IX, 134 pages. 1995.

Vol. $m$ 29: C. Bendjaballah, Introduction to Photon Communication. VII, 193 pages. 1995.

Vol. m 30: A. J. Greer, W. J. Kossler, Low Magnetic Fields in Anisotropic Superconductors. VII, 161 pages. 1995.

Vol. m 31 (Corr. Second Printing): P. Busch, M. Grabowski, P.J. Lahti, Operational Quantum Physics. XII, 230 pages. 1997.

Vol. m 32: L. de Broglie, Diverses questions de mécanique et de thermodynamique classiques et relativistes. XII, 198 pages. 1995.

Vol. m 33: R. Alkofer, H. Reinhardt, Chiral Quark Dynamics. VIII, 115 pages. 1995.
Vol. $m$ 34: R. Jost, Das Märchen vom Elfenbeinernen Turm. VIII, 286 pages. 1995.

Vol. $m$ 35: E. Elizalde, Ten Physical Applications of Spectral Zeta Functions. XIV, 224 pages. 1995.

Vol. $m$ 36: G. Dunne, Self-Dual Chern-Simons Theories. $\mathrm{X}, 217$ pages. 1995.

Vol. m 37: S. Childress, A.D. Gilbert, Stretch, Twist, Fold: The Fast Dynamo. XI, 406 pages. 1995.

Vol. m 38: J. González, M. A. Martín-Delgado, G. Sierra, A. H. Vozmediano, Quantum Electron Liquids and High-Tc Superconductivity. X, 299 pages. 1995.

Vol. $m$ 39: L. Pittner, Algebraic Foundations of NonCom-mutative Differential Geometry and Quantum Groups. XII, 469 pages. 1996.

Vol. m 40: H.-J. Borchers, Translation Group and Particle Representations in Quantum Field Theory. VII, 131 pages. 1996.

Vol. In 41: B. K. Chakrabarti, A. Dutta, P. Sen, Quantum Ising Phases and Transitions in Transverse Ising Models. X, 204 pages. 1996.

Vol. m 42: P. Bouwknegt, J. McCarthy, K. Pilch, The W3 Algebra. Modules, Semi-infinite Cohomology and BV Algebras. XI, 204 pages. 1996.

Vol. $m$ 43: M. Schottenloher, A Mathematical Introduction to Conformal Field Theory. VIII, 142 pages. 1997.

Vol. m 44: A. Bach, Indistinguishable Classical Particles. VIII, 157 pages. 1997.

Vol. m 45: M. Ferrari, V. T. Granik, A. Imam, J. C. Nadeau (Eds.), Advances in Doublet Mechanics. XVI, 214 pages. 1997.

Vol. $\mathrm{m}$ 46: M. Camenzind, Les noyaux actifs de galaxies. XVIII, 218 pages. 1997.

Vol. $m$ 47: L. M. Zubov, Nonlinear Theory of Dislocations and Disclinations in Elastic Body. VI, 205 pages. 1997.

Vol. $m$ 48: P. Kopietz, Bosonization of Interacting Fermions in Arbitrary Dimensions. XII, 259 pages. 1997.

Vol. m 49: M. Zak, J. B. Zbilut, R. E. Meyers, From Instability to Intelligence. Complexity and Predictability in Nonlinear Dynamics. XIV, 552 pages. 1997.

Vol. m 50: J. Ambjørn, M. Carfora, A. Marzuoli, The Geometry of Dynamical Triangulations. VI, 197 pages. 1997.

Vol. m 51: G. Landi, An Introduction to Noncommutative Spaces and Their Geometries. XI, 200 pages. 1997.

Vol. $m$ 52: M. Hénon, Generating Families in the Restricted Three-Body Problem. XI, 278 pages. 1997.

Vol. m 53: M. Gad-el-Hak, A. Pollard, J.-P. Bonnet (Eds.), Flow Control. Fundamentals and Practices. XII, 527 pages. 1998.

Vol, m 54: Y. Suzuki, K. Varga, Stochastic Variational Approach to Quantum-Mechanical Few-Body Problems. XIV, 324 pages. 1998.

Vol. m 55: F. Busse, S. C. Müller, Evolution of Spontaneous Structures in Dissipative Continuous Systems. $\mathrm{X}, 559$ pages. 1998 . 
ISSN 0075-8450

ISBN 3-540-65702-9

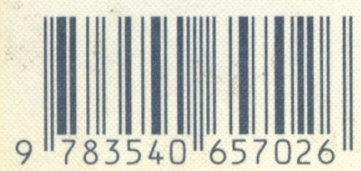

\section{http://www.springer.de}

